\title{
Algorithms for the ROD DSP of the ATLAS Hadronic Tile Calorimeter
}

\author{
J. Castelo ${ }^{\text {a }, ~ V . ~ C a s t i l l o ~}{ }^{\text {a }}$ C. Cuenca ${ }^{\mathrm{a}}$, A. Ferrer ${ }^{\mathrm{a}}$, E. Fullana ${ }^{\mathrm{a}}$, V. González ${ }^{\mathrm{b}}$, \\ E. Higon ${ }^{\mathrm{a}}$, A. Munar ${ }^{\mathrm{a}}$, J. Poveda ${ }^{\mathrm{a}}$, A. Ruiz-Martínez ${ }^{\mathrm{a}}$, B. Salvachua ${ }^{\mathrm{a}}$, \\ E. Sanchís ${ }^{\mathrm{b}}$, C. Solans ${ }^{\mathrm{a}}$, J. Soret ${ }^{\mathrm{b}}$, J. Torres ${ }^{\mathrm{b}}$, A. Valero ${ }^{\mathrm{a}}$, J. A. Valls ${ }^{\mathrm{a}}$ \\ a Departamento de Física Atómica, Molecular y Nuclear and IFIC, \\ Aptdo. 22085, Valencia, España \\ ${ }^{\mathrm{b}}$ Departamento de Electrónica, Universidad de Valencia, España
}

belen.salvachua@ific.uv.es

\begin{abstract}
In this paper we present the performance of two algorithms currently running in the Tile Calorimeter Read Out Driver boards for the commissioning of ATLAS. The first algorithm presented is the so called Optimal Filtering. It reconstructs the deposited energy in the Tile Calorimeter and the arrival time of the data. The second algorithm is the MTag which tags low transverse momentum muons that may escape the ATLAS muon spectrometer first level trigger.

Comparisons between online (inside the Read Out Drivers) and offline implementations are done showing an agreement better than $95 \%$ for the Optimal Filtering algorithm and up to $85 \%$ for the MTag algorithm. The processing time was measured for both algorithms running together with a resulting time of $29.4 \mu$ s which, although above the $10 \mu$ s of the first level trigger, it fulfills the requirements of the commissioning trigger $(\sim 1 \mathrm{~Hz})$. We expect further optimizations of the algorithms which will reduce their processing time below $10 \mu \mathrm{s}$.
\end{abstract}

\section{INTRODUCTION}

The ATLAS detector (see Figure 1) is a general purpose experiment designed to exploit the physics discovery potential of the next proton-proton collider at CERN, the Large Hadron Collider (LHC).

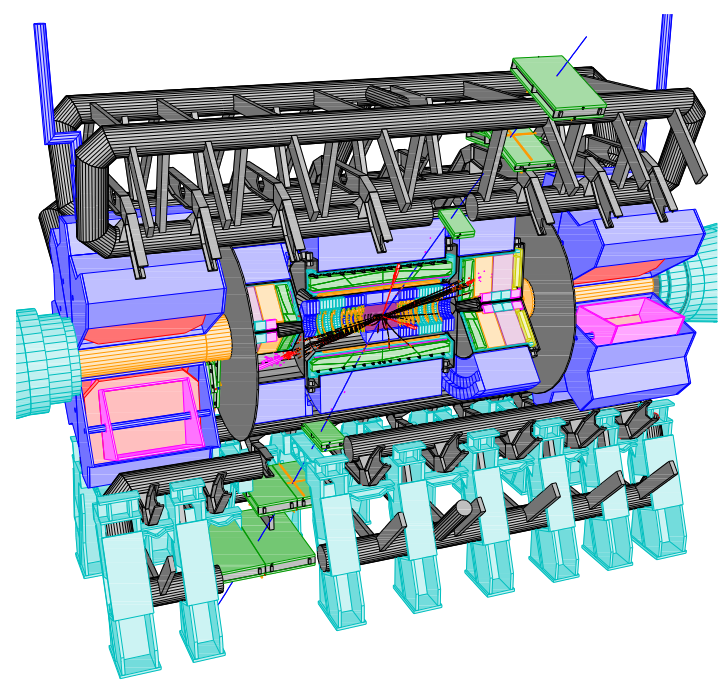

Figure 1: ATLAS detector.
The ATLAS hadronic Tile Calorimeter (TileCal) [1] is a sampling calorimeter made out of iron as absorber material and plastic scintillating plates as active medium. The light produced in the scintillating tiles is collected through wavelength shifting fibers and read-out by photomultipliers. The analogue electrical signal of the photomultipliers is digitized in samples of 10 bits taken each $25 \mathrm{~ns}$, the latency between two consecutive LHC bunch crossings. Therefore, the $\sim 10000$ photomultipliers of the calorimeter produce up to $\sim 164 \mathrm{Gbps}$ of data that is readout by the TileCal back-end electronics.

The main element of the back-end electronics is the Read Out System [2]. This system is composed of 32 Read Out Driver (ROD) boards (see Figure 2). The RODs are based on a custom 9U VME64x board equipped with up to four Processing Units (PUs) which are pluggable daughter-boards. The PUs process the data coming from TileCal front-end to feed the second level trigger with information of energy deposited in the calorimeter and other relevant quantities.

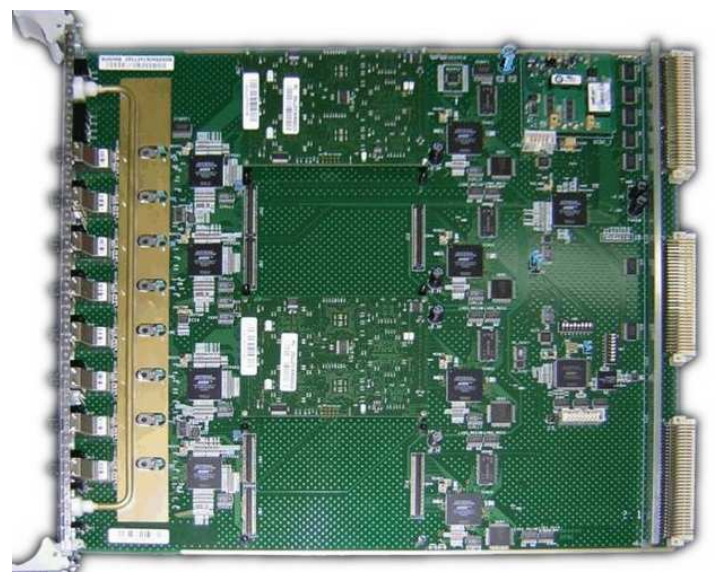

Figure 2: ROD motherboard.

The PUs are equipped with the TMS320C6414 $\mathrm{x}^{\mathrm{TM}}$ Digital Signal Processors (DSPs) of Texas Instruments in order to execute reconstruction algorithms in real time. The latency of the second level trigger is $10 \mu \mathrm{s}$ which sets stringent requirements on the processing time of the reconstruction algorithms.

In this paper we present the performance of two algorithms implemented in the DSPs. We propose an algorithm for 
the energy reconstruction, the so called Optimal Filtering (OF) [3], which reconstructs the amplitude and time of the photomultiplier signal by means of weighted sums of the digital samples. Additionally, we propose a fast muon tag algorithm, named MTag, to identify low transverse momentum $\left(p_{\mathrm{T}}\right)$ muons that may escape the muon spectrometer. The tagging of these muons helps to trigger interesting B-physics channels.

Both algorithms have been tested online in the ATLAS commissioning with cosmic rays runs. The performance of both algorithms and the processing time required is presented in this paper.

\section{EXPERIMENTAL SETUP}

Until 2007 the ATLAS detector is being commissioned in the ATLAS cavern. During this period all subdetectors will be progressively integrated into the final ATLAS Trigger and Data Acquisition system.

During 2005 and 2006, TileCal has been fully assembled in the underground experimental hall UX15. A program of cosmic rays data acquisition has been planned for TileCal in standalone mode. The trigger signal was provided by the TileCal trigger electronics, using custom coincidence boards which take as input up to 192 TileCal trigger tower analog signals [4]; half in the top part and half in the bottom part. A signal coincidence in the upper and lower part of the calorimeter triggers the data acquisition. (see Figure 3).

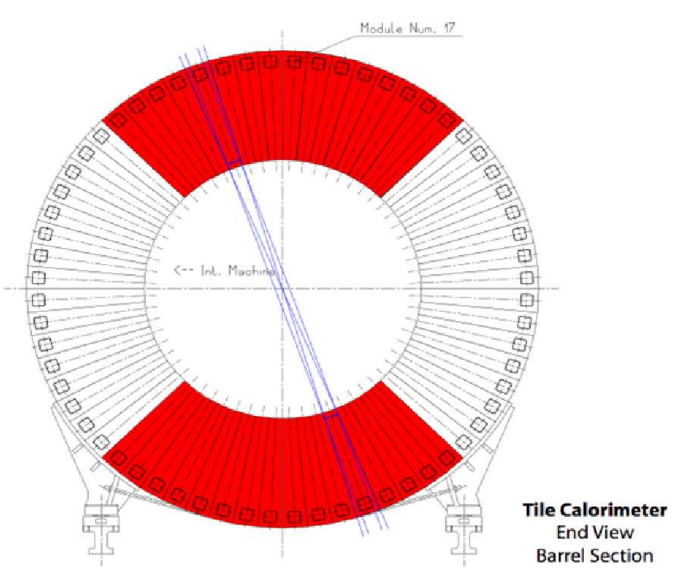

Figure 3: TileCal cosmic muon trigger.

The cosmic muon trigger is not synchronized with the data acquisition system and thus the arrival time of the events follows a flat distribution in a wide time window.

Our goal is to satisfactorily reconstruct the energy deposited by the muons and to check the efficiency of the MTag algorithm.

\section{Algorithms}

Both the Optimal Filtering and the MTag algorithms are implemented in the ROD DSPs which are the Texas Instruments TMS320C6414x ${ }^{\mathrm{TM}}$ DSPs. They are fixed-point processors which perform up to 32-bit data multiply and accumulate instructions. Their CPU contains only multiplier and ALU units; therefore divisions are not allowed and they are implemented as shift instructions, to divide in powers of 2 , and with the use of look-up tables to implement more complex operations. Consequently the algorithms inside the DSP should reach a compromise between simplicity and accuracy.

\section{A. Optimal Filtering}

The Optimal Filtering algorithm reconstructs the amplitude and phase of a digitized signal by a linear combination of its digital samples, pedestal subtracted (see Eq. 1 and Eq. 2):

$$
\begin{gathered}
A=\sum_{i=1}^{n} a_{i}\left(S_{i}-p\right), \\
\tau=\frac{1}{A} \sum_{i=1}^{n} b_{i}\left(S_{i}-p\right),
\end{gathered}
$$

where $S_{i}$ represents the digital sample $i$ and $n$ is the total number of samples. We define the pedestal, $p$, as the baseline of the signal. The amplitude, $A$, is the distance from the pedestal to the peak and the phase, $\tau$, is defined as the time between the central sample and the peak of the pulse (see Figure 4).

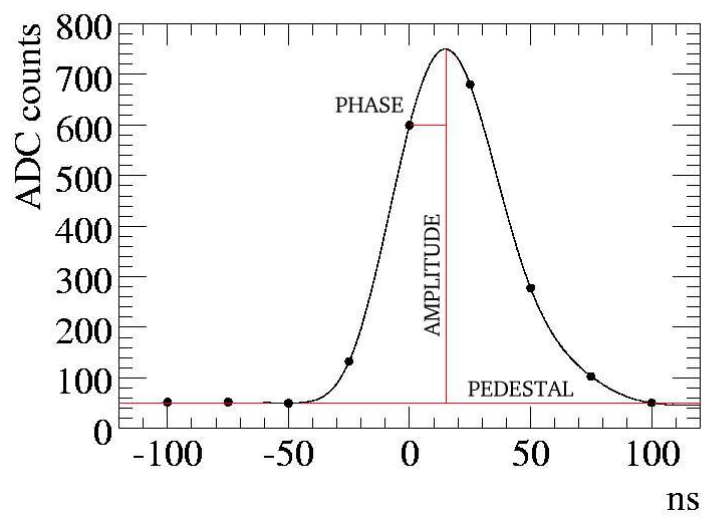

Figure 4: Definition of amplitude, phase and pedestal.

The weights, $\boldsymbol{a}$ and $\boldsymbol{b}$, are obtained from the pulse shape of the photomultipliers and the noise autocorrelation matrix. The process to calculate them minimizes the effect of the noise in the amplitude and time reconstruction [5].

These weights are calculated assuming small phases, which means that increasing $\tau$ the quality of the reconstruction decreases. This occurs during the TileCal commissioning since the data is not synchronized with the trigger clock. In order to properly calculate the energy deposited in the calorimeter during the commissioning phase we introduce one iteration in the $\mathrm{OF}$ algorithm to estimate the arrival time of the data, although this increases the processing time. Anyway, the expected trigger rate is $\sim 1 \mathrm{~Hz}$ which slows down the time restrictions.

During the first iteration, the amplitude and the phase are calculated assuming that the peak is close to the central sample, phase equal to 0 ns (see Eq. 3 and Eq. 4): 


$$
\begin{gathered}
A_{1}=\left.\sum_{i=1}^{n} a_{i}\right|_{\tau=0}\left(S_{i}-p\right), \\
\tau_{1}=\left.\frac{1}{A_{1}} \sum_{i=1}^{n} b_{i}\right|_{\tau=0}\left(S_{i}-p\right) .
\end{gathered}
$$

This iteration gives a first approximation of the phase. In the second iteration the amplitude and the phase are again calculated assuming that the peak is now around $\tau_{1}$ (see Eq. 5 and Eq. 6):

$$
\begin{gathered}
A_{2}=\left.\sum_{i=1}^{n} a_{i}\right|_{\tau=\tau_{1}}\left(S_{i}-p\right), \\
\tau_{2}=\left.\frac{1}{A_{2}} \sum_{i=1}^{n} b_{i}\right|_{\tau=\tau_{1}}\left(S_{i}-p\right) .
\end{gathered}
$$

Weights calculated for phases between $-15 \mathrm{~ns}$ and $15 \mathrm{~ns}$ are stored in the ROD DSPs memory in order to use them during the iteration mechanism. The result of the second iteration, $A_{2}$ and $\tau_{2}$, is used as input by the MTag algorithm.

\section{B. Muon Tag}

The primary goal of the MTag algorithm [6] [7] is to search for muons taking into account the energy deposited in each layer of TileCal. Figure 5 shows the TileCal cell structure with 3 layers (A, BC and D cells) and with a projective geometry in $\eta$. In order to identify the muons, the typical energy deposition in each cell is limited by a high and a low threshold:

$$
t h r_{i}^{\text {low }} \leq E_{i} \leq t h r_{i}^{\text {high }} \quad i=1,2,3
$$

If this condition is fulfilled in each of the 3 layers with a projective pattern in $\eta$, the muon is tagged. In order to be efficient on events in where the muon loses a considerable fraction of its energy in one of the layers, muons are also tagged if Eq. 7 is fulfilled in two layers while in the third layer the energy deposition is above the low threshold.

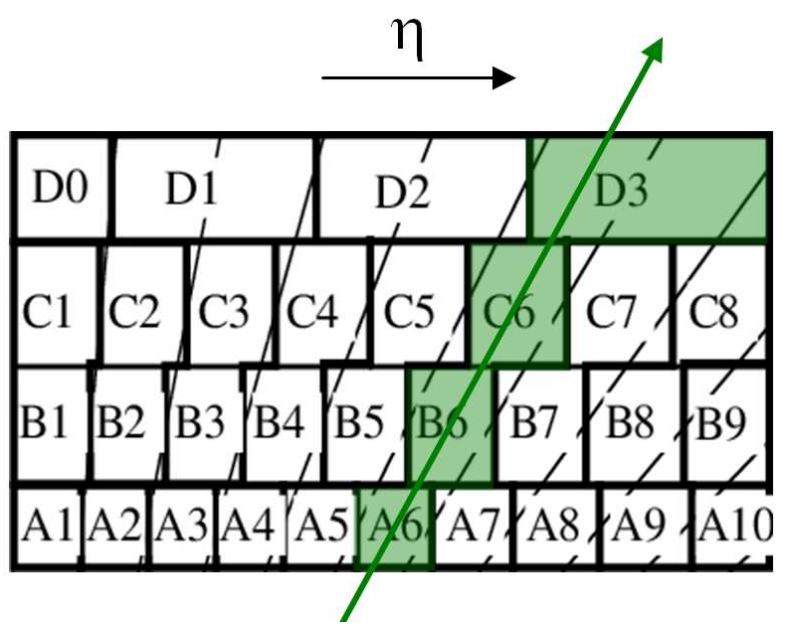

Figure 5: Muon path going through a tower in $\eta$.
The low energy threshold is meant to cut electronic noise and minimum bias pile-up events. In this algorithm, all cells have the same low energy threshold values. The high energy thresholds are meant to delimit the maximum muon energy deposition while eliminating hadronic showers and tails. These thresholds are determined for each individual cell, depending on the pseudorapidity of the muon trajectory.

The result of the MTag algorithm is the number of muons inside a TileCal module and the pseudorapidity where they were found.

\section{RESULTS}

During July and August 2006 several runs of cosmic rays were acquired and reconstructed online in the ROD DSPs. Figure 6 shows the histogram of the reconstructed amplitude in the ROD DSPs (online) and the offline reconstruction for all the operative channels during the acquisition. The large tail corresponds to energy deposited by cosmic muons, while the peak around zero corresponds mostly to electronic noise. The agreement between both reconstructions is larger than $99 \%$ for amplitudes above $3 \sigma$ of the noise. For amplitudes smaller than $3 \sigma$ the difference between the online and offline amplitude reconstruction is expected to be larger. Eq. 4 shows that in order to calculate the phase at the first iteration we need to divide by the amplitude. In the ROD DSPs, this implementation is carried out by the use of a look-up table which decreases the resolution of the phase for amplitudes close to zero.

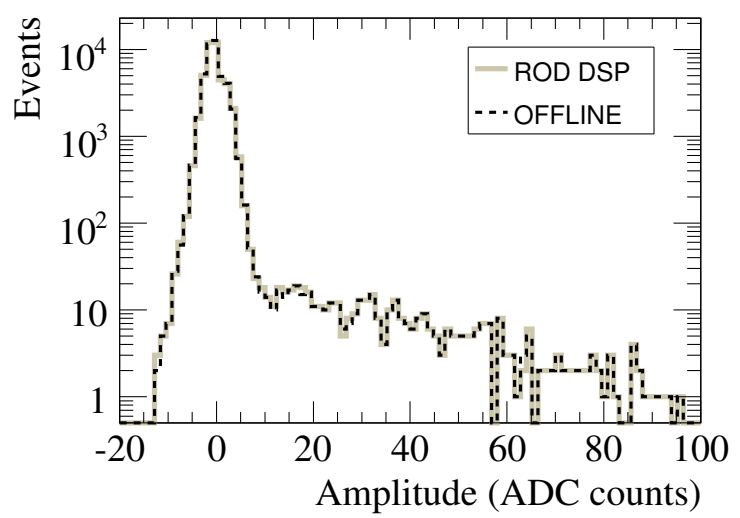

Figure 6: Reconstructed amplitude using Optimal Filtering, offline and inside the ROD DSPs.

Figure 7 shows the online and offline phase reconstruction for amplitudes larger than $3 \sigma$. The error of the phase reconstruction is larger than the error of the amplitude. This is due again to the use of look-up tables. The discrepancy with the offline result is $\sim 5 \%$.

Figure 8 shows the energy distribution of the online tagged muons in the ROD DSPs using Optimal Filtering for the energy reconstruction. The results are compared with the distributions of the offline MTag algorithm using the Fit method for the energy reconstruction which is the standard offline algorithm. 
Even though the energy taken as input of the offline MTag is slightly different from the online, up to $85 \%$ of the offline tagged muons are also tagged in the ROD DSPs.

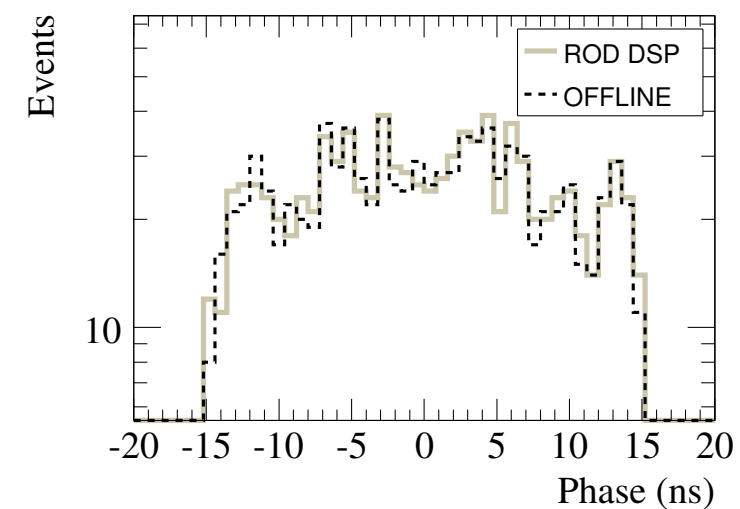

Figure 7: Reconstructed phase using Optimal Filtering, offline and inside the ROD DSPs.

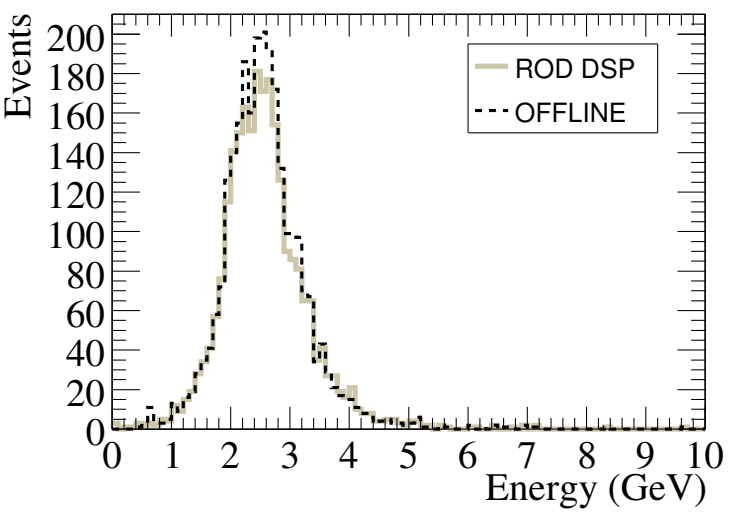

Figure 8: Energy distribution of the tagged muons, offline and inside the ROD DSPs.

The processing time of the online algorithms is a very important issue, since the latency of the first level trigger is $10 \mu \mathrm{s}$. However this maximum rate will not be achieved during the first years of the LHC operation and the rate of the cosmic muons acquisition is $\sim 1 \mathrm{~Hz}$.

The DSP processing time has been measured in our laboratory (see Table 1). Globally these times are well above to $10 \mu \mathrm{s}$, however, the current implementation is coded in $\mathrm{C}$ and a great improvement is expected after migrating to assembler. Furthermore, the $\mathrm{OF}$ algorithm is adapted to the commissioning conditions and makes use of iterations. Nevertheless, in the final configuration, when timing is fixed with the LHC clock no iterations will be needed and the processing time will be reduced.
Table 1: ROD DSP processing time.

\begin{tabular}{cc}
\hline Algorithm & Time $(\mu \mathrm{s})$ \\
\hline OF & 27.1 \\
OF and MTag & 29.4 \\
\hline
\end{tabular}

\section{Conclusions}

During July and August 2006 the Optimal Filtering and the MTag algorithms were tested within the ATLAS commissioning environment. The results of their implementation in the ROD DSPs (online) and offline were shown. For the Optimal Filtering algorithm the agreement with the offline reconstruction was better that $99 \%$ for events with amplitudes larger than $3 \sigma$ of the noise; for the phase reconstruction an agreement of $95 \%$ was found. The decrease of the online resolution was due to the use of look-up tables in order to implement the division by the amplitude. The results of the MTag algorithm analysis showed that up to $85 \%$ of the offline tagged muons were also tagged in the ROD DSPs, even though the reconstruction algorithms were different.

The processing time was also measured for both algorithms running together with a result of $29.4 \mu \mathrm{s}$ which is larger than the LHC final requirements $(10 \mu \mathrm{s})$ but it still fulfills the requirements of the ATLAS commissioning which is $\sim 1 \mathrm{~Hz}$.

\section{REFERENCES}

[1] ATLAS Collaboration, Tile Calorimeter Technical Design Report, Technical Report CERN/LHCC 96-42, CERN, 1996.

[2] J. Castelo et al., TileCal ROD Hardware and Software Requirements, ATLAS Internal Note, CERN-ATL-TILECAL-2005-003, 2005.

[3] E. Fullana et al., Optimal Filtering in the ATLAS Hadronic Tile Calorimeter, ATLAS Internal Note, CERN-ATL-TILECAL-2005-001, 2005.

[4] K. Anderson et al., Stand-alone cosmic ray trigger electronics for the ATLAS Tile Calorimeter, 10th Workshop on electronics for LHC and future experiments, pages 327-331, 2004.

[5] W.E.Cleland, E.G. Stern, Signal processing considerations for liquid ionization calorimeters in high rate environment, Nucl. Instrum. Meth. A 338, 467 (2004).

[6] A. Ruiz-Martínez, Development of a low $p_{\mathrm{T}}$ muon LVL2 trigger algorithm with the ATLAS TileCal detector, Master's Thesis, Universidad de Valencia, September 2006.

[7] G. Usai [ATLAS Collaboration], Trigger of low $p_{\mathrm{T}}$ muons with the ATLAS hadronic calorimeter, Nucl. Instrum. Meth. A 518, 36 (2004). 